\title{
Fractional Sums and Differences with Binomial Coefficients
}

\author{
Thabet Abdeljawad, ${ }^{1}$ Dumitru Baleanu, ${ }^{1,2,3}$ Fahd Jarad, ${ }^{1}$ and Ravi P. Agarwal ${ }^{4}$ \\ ${ }^{1}$ Department of Mathematics, Faculty of Art and Sciencs, Çankaya University, Balgat, 06530 Ankara, Turkey \\ ${ }^{2}$ Institute of Space Sciences, P.O. BOX MG-23, 76900 Magurele-Bucharest, Romania \\ ${ }^{3}$ Department of Chemical and Materials Engineering, Faculty of Engineering, King Abdulaziz University, \\ P.O. Box 80204, Jeddah 21589, Saudi Arabia \\ ${ }^{4}$ Department of Mathematics, Texas A \& M University, 700 University Boulevard, Kingsville, TX, USA
}

Correspondence should be addressed to Thabet Abdeljawad; tabdeljawad@psu.edu.sa

Received 31 March 2013; Accepted 3 May 2013

Academic Editor: Shurong Sun

Copyright @ 2013 Thabet Abdeljawad et al. This is an open access article distributed under the Creative Commons Attribution License, which permits unrestricted use, distribution, and reproduction in any medium, provided the original work is properly cited.

\begin{abstract}
In fractional calculus, there are two approaches to obtain fractional derivatives. The first approach is by iterating the integral and then defining a fractional order by using Cauchy formula to obtain Riemann fractional integrals and derivatives. The second approach is by iterating the derivative and then defining a fractional order by making use of the binomial theorem to obtain Grünwald-Letnikov fractional derivatives. In this paper we formulate the delta and nabla discrete versions for left and right fractional integrals and derivatives representing the second approach. Then, we use the discrete version of the Q-operator and some discrete fractional dual identities to prove that the presented fractional differences and sums coincide with the discrete Riemann ones describing the first approach.
\end{abstract}

\section{Introduction and Preliminaries}

Fractional calculus (FC) is developing very fast in both theoretical and applied aspects. As a result, FC is used intensively and successfully in the last few decades to describe the anomalous processes which appear in complex systems [1-6]. Very recently, important results in the field of fractional calculus and its applications were reported (see e.g., [7-10] and the references therein). The complexity of the real world phenomena is a great source of inspiration for the researchers to invent new fractional tools which will be able to dig much dipper into the mysteries of the mother nature. Historically the FC passed through different periods of evolutions, and it started to face very recently a new provocation: how to formulate properly its discrete counterpart [11-24]. At this stage, we have to stress on the fact that in the classical discrete equations their roots are based on the functional difference equations, therefore, the natural question is to find the generalization of these equations to the fractional case. In other words, we will end up with generalizations of the basic operators occurring in standard difference equations. As it was expected, there were several attempts to do this generalization as well as to apply this new techniques to investigate the dynamics of some complex processes. In recent years, the discrete counterpart of the fractional Riemann-Liouville, Caputo, was investigated mainly thinking how to apply techniques from the time scales calculus to the expressions of the fractional operators. Despite of the beauty of the obtained results, one simple question arises: can we obtain the same results from a new point of view which is more simpler and more intuitive? Having all above mentioned thinks in mind we are going to use the binomial theorem in order to get Grünwald-Letnikov fractional derivatives. After that, we proved that the results obtained coincide with the ones obtained by the discretization of the Riemann-Liouville operator. In this manner, we believe that it becomes more clear what the fractional difference equations bring new in description of the related complex phenomena described.

For a natural number $n$, the fractional polynomial is defined by

$$
t^{(n)}=\prod_{j=0}^{n-1}(t-j)=\frac{\Gamma(t+1)}{\Gamma(t+1-n)}
$$


where $\Gamma$ denotes the special gamma function and the product is zero when $t+1-j=0$ for some $j$. More generally, for arbitrary $\alpha$, define

$$
t^{(\alpha)}=\frac{\Gamma(t+1)}{\Gamma(t+1-\alpha)},
$$

where the convention of that division at pole yields zero. Given that the forward and backward difference operators are defined by

$$
\Delta f(t)=f(t+1)-f(t), \quad \nabla f(t)=f(t)-f(t-1),
$$

respectively, we define iteratively the operators $\Delta^{m}=\Delta\left(\Delta^{m-1}\right)$ and $\nabla^{m}=\nabla\left(\nabla^{m-1}\right)$, where $m$ is a natural number.

Here are some properties of the factorial function.

Lemma 1 (see [13]). Assume the following factorial functions are well defined.

(i) $\Delta t^{(\alpha)}=\alpha t^{(\alpha-1)}$.

(ii) $(t-\mu) t^{(\mu)}=t^{(\mu+1)}$, where $\mu \in \mathbb{R}$.

(iii) $\mu^{(\mu)}=\Gamma(\mu+1)$.

(iv) If $t \leq r$, then $t^{(\alpha)} \leq r^{(\alpha)}$ for any $\alpha>r$.

(v) If $0<\alpha<1$, then $t^{(\alpha \nu)} \geq\left(t^{(\nu)}\right)^{\alpha}$.

(vi) $t^{(\alpha+\beta)}=(t-\beta)^{(\alpha)} t^{(\beta)}$.

Also, for our purposes we list down the following two properties, the proofs of which are straightforward:

$$
\begin{gathered}
\nabla_{s}(s-t)^{(\alpha-1)}=(\alpha-1)(\rho(s)-t)^{(\alpha-2)} \\
\nabla_{t}(\rho(s)-t)^{(\alpha-1)}=-(\alpha-1)(\rho(s)-t)^{(\alpha-2)} .
\end{gathered}
$$

For the sake of the nabla fractional calculus, we have the following definition.

Definition 2 (see [25-28]). (i) For a natural number $m$, the $m$ rising (ascending) factorial of $t$ is defined by

$$
t^{\bar{m}}=\prod_{k=0}^{m-1}(t+k), \quad t^{\overline{0}}=1
$$
by

(ii) For any real number, the $\alpha$ rising function is defined

$$
t^{\bar{\alpha}}=\frac{\Gamma(t+\alpha)}{\Gamma(t)}, \quad t \in \mathbb{R}-\{\ldots,-2,-1,0\}, \quad 0^{\alpha}=0 .
$$

Regarding the rising factorial function, we observe the following:

(i)

$$
\nabla\left(t^{\bar{\alpha}}\right)=\alpha t^{\overline{\alpha-1}}
$$

(ii)

$$
\left(t^{\bar{\alpha}}\right)=(t+\alpha-1)^{(\alpha)},
$$

(iii)

$$
\Delta_{t}(s-\rho(t))^{\bar{\alpha}}=-\alpha(s-\rho(t))^{\overline{\alpha-1}} .
$$

Notation:

(i) For a real $\alpha>0$, we set $n=[\alpha]+1$, where $[\alpha]$ is the greatest integer less than $\alpha$.

(ii) For real numbers $a$ and $b$, we denote $\mathbb{N}_{a}=\{a, a+1, \ldots\}$ and ${ }_{b} \mathbb{N}=\{b, b-1, \ldots\}$.

(iii) For $n \in \mathbb{N}$ and real $a$, we denote

$$
{ }_{\Theta} \Delta^{n} f(t) \triangleq(-1)^{n} \Delta^{n} f(t) \text {. }
$$

(iv) For $n \in \mathbb{N}$ and real $b$, we denote

$$
\nabla_{\odot}^{n} f(t) \triangleq(-1)^{n} \nabla^{n} f(t) .
$$

The following definition and the properties followed can be found in [29] and the references therein.

Definition 3 (see [29]). Let $\sigma(t)=t+1$ and $\rho(t)=t-1$ be the forward and backward jumping operators, respectively. Then

(i) the (delta) left fractional sum of order $\alpha>0$ (starting from $a$ ) is defined by

$$
\Delta_{a}^{-\alpha} f(t)=\frac{1}{\Gamma(\alpha)} \sum_{s=a}^{t-\alpha}(t-\sigma(s))^{(\alpha-1)} f(s), \quad t \in \mathbb{N}_{a+\alpha} .
$$

(ii) The (delta) right fractional sum of order $\alpha>0$ (ending at $b$ ) is defined by

$$
\begin{aligned}
{ }_{b} \Delta^{-\alpha} f(t) & =\frac{1}{\Gamma(\alpha)} \sum_{s=t+\alpha}^{b}(s-\sigma(t))^{(\alpha-1)} f(s) \\
& =\frac{1}{\Gamma(\alpha)} \sum_{s=t+\alpha}^{b}(\rho(s)-t)^{(\alpha-1)} f(s), \quad t \in_{b-\alpha} \mathbb{N} .
\end{aligned}
$$

(iii) The (nabla) left fractional sum of order $\alpha>0$ (starting from $a$ ) is defined by

$\nabla_{a}^{-\alpha} f(t)=\frac{1}{\Gamma(\alpha)} \sum_{s=a+1}^{t}(t-\rho(s))^{\overline{\alpha-1}} f(s), \quad t \in \mathbb{N}_{a+1}$.

(iv) The (nabla) right fractional sum of order $\alpha>0$ (ending at $b$ ) is defined by

${ }_{b} \nabla^{-\alpha} f(t)=\frac{1}{\Gamma(\alpha)} \sum_{s=t}^{b-1}(s-\rho(t))^{\overline{\alpha-1}} f(s)$

$$
=\frac{1}{\Gamma(\alpha)} \sum_{s=t}^{b-1}(\sigma(s)-t)^{\overline{\alpha-1}} f(s), \quad t \in_{b-1} \mathbb{N} .
$$


Regarding the delta left fractional sum, we observe the following:

(i) $\Delta_{a}^{-\alpha}$ maps functions defined on $\mathbb{N}_{a}$ to functions defined on $\mathbb{N}_{a+\alpha}$.

(ii) $u(t)=\Delta_{a}^{-n} f(t), n \in \mathbb{N}$, satisfies the initial value problem:

$$
\begin{gathered}
\Delta^{n} u(t)=f(t), \quad t \in N_{a}, \\
u(a+j-1)=0, \quad j=1,2, \ldots, n .
\end{gathered}
$$

(iii) The Cauchy function $(t-\sigma(s))^{(n-1)} /(n-1)$ ! vanishes at $s=t-(n-1), \ldots, t-1$.

Regarding the delta right fractional sum, we observe the following:

(i) ${ }_{b} \Delta^{-\alpha}$ maps functions defined on ${ }_{b} \mathbb{N}$ to functions defined on ${ }_{b-\alpha} \mathbb{N}$.

(ii) $u(t)={ }_{b} \Delta^{-n} f(t), n \in \mathbb{N}$, satisfies the initial value problem:

$$
\begin{gathered}
\nabla_{\ominus}^{n} u(t)=f(t), \quad t \in{ }_{b} N, \\
u(b-j+1)=0, \quad j=1,2, \ldots, n .
\end{gathered}
$$

(iii) The Cauchy function $(\rho(s)-t)^{(n-1)} /(n-1)$ ! vanishes at $s=t+1, t+2, \ldots, t+(n-1)$.

Regarding the nabla left fractional sum, we observe the following:

(i) $\nabla_{a}^{-\alpha}$ maps functions defined on $\mathbb{N}_{a}$ to functions defined on $\mathbb{N}_{a}$.

(ii) $\nabla_{a}^{-n} f(t)$ satisfies the $n$ th-order discrete initial value problem:

$\nabla^{n} y(t)=f(t), \quad \nabla^{i} y(a)=0, \quad i=0,1, \ldots, n-1$.

(iii) The Cauchy function $(t-\rho(s))^{\overline{n-1}} / \Gamma(n)$ satisfies $\nabla^{n} y(t)=0$.

Regarding the nabla right fractional sum we observe the following:

(i) ${ }_{b} \nabla^{-\alpha}$ maps functions defined on ${ }_{b} \mathbb{N}$ to functions defined on ${ }_{b} \mathbb{N}$.

(ii) ${ }_{b} \nabla^{-n} f(t)$ satisfies the $n$ th-order discrete initial value problem:

${ }_{\ominus} \Delta^{n} y(t)=f(t), \quad{ }_{\ominus} \Delta^{i} y(b)=0, \quad i=0,1, \ldots, n-1$.

The proof can be done inductively. Namely, assuming it is true for $n$, we have

${ }_{\ominus} \Delta^{n+1}{ }_{b} \nabla^{-(n+1)} f(t)={ }_{\ominus} \Delta^{n}\left[-\Delta_{b} \nabla^{-(n+1)} f(t)\right]$.

By the help of (9), it follows that

$$
{ }_{\ominus} \Delta^{n+1}{ }_{b} \nabla^{-(n+1)} f(t)={ }_{\ominus} \Delta^{n}{ }_{b} \nabla^{-n} f(t)=f(t) \text {. }
$$

The other part is clear by using the convention that $\sum_{k=s+1}^{s}=0$. (iii) The Cauchy function $(s-\rho(t))^{\overline{n-1}} / \Gamma(n)$ satisfies ${ }_{\ominus} \Delta^{n} y(t)=0$.

Definition 4. (i) [12] The (delta) left fractional difference of order $\alpha>0$ (starting from $a$ ) is defined by

$$
\begin{aligned}
\Delta_{a}^{\alpha} f(t) & =\Delta^{n} \Delta_{a}^{-(n-\alpha)} f(t) \\
& =\frac{\Delta^{n}}{\Gamma(n-\alpha)} \sum_{s=a}^{t-(n-\alpha)}(t-\sigma(s))^{(n-\alpha-1)} f(s), \\
& t \in \mathbb{N}_{a+(n-\alpha)} .
\end{aligned}
$$

(ii) [19] The (delta) right fractional difference of order $\alpha>$ 0 (ending at $b$ ) is defined by

$$
\begin{aligned}
&{ }_{b} \Delta^{\alpha} f(t)=\nabla_{\odot b}^{n} \Delta^{-(n-\alpha)} f(t) \\
&=\frac{(-1)^{n} \nabla^{n}}{\Gamma(n-\alpha)} \sum_{s=t+(n-\alpha)}^{b}(s-\sigma(t))^{(n-\alpha-1)} f(s), \\
& t \in_{b-(n-\alpha)} \mathbb{N} .
\end{aligned}
$$

(iii) [20] The (nabla) left fractional difference of order $\alpha>$ 0 (starting from $a$ ) is defined by

$$
\begin{aligned}
\nabla_{a}^{\alpha} f(t) & =\nabla^{n} \nabla_{a}^{-(n-\alpha)} f(t) \\
& =\frac{\nabla^{n}}{\Gamma(n-\alpha)} \sum_{s=a+1}^{t}(t-\rho(s))^{\overline{n-\alpha-1}} f(s), \\
& t \in \mathbb{N}_{a+1} .
\end{aligned}
$$

(iv) $[29,30]$ The (nabla) right fractional difference of order $\alpha>0$ (ending at $b$ ) is defined by

$$
\begin{aligned}
&{ }_{b} \nabla^{\alpha} f(t)={ }_{\Theta} \Delta^{n}{ }_{b} \nabla^{-(n-\alpha)} f(t) \\
&=\frac{(-1)^{n} \Delta^{n}}{\Gamma(n-\alpha)} \sum_{s=t}^{b-1}(s-\rho(t))^{\overline{n-\alpha-1}} f(s), \\
& t \in{ }_{b-1} \mathbb{N} .
\end{aligned}
$$

Regarding the domains of the fractional type differences we observe the following.

(i) The delta left fractional difference $\Delta_{a}^{\alpha}$ maps functions defined on $\mathbb{N}_{a}$ to functions defined on $\mathbb{N}_{a+(n-\alpha)}$.

(ii) The delta right fractional difference ${ }_{b} \Delta^{\alpha}$ maps functions defined on ${ }_{b} \mathbb{N}$ to functions defined on ${ }_{b-(n-\alpha)} \mathbb{N}$.

(iii) The nabla left fractional difference $\nabla_{a}^{\alpha}$ maps functions defined on $\mathbb{N}_{a}$ to functions defined on $\mathbb{N}_{a+n}$.

(iv) The nabla right fractional difference ${ }_{b} \nabla^{\alpha}$ maps functions defined on ${ }_{b} \mathbb{N}$ to functions defined on ${ }_{b-n} \mathbb{N}$. 
Lemma 5 (see [15]). Let $0 \leq n-1<\alpha \leq n$, and let $y(t)$ be defined on $\mathbb{N}_{a}$. Then the following statements are valid:

(i) $\left(\Delta_{a}^{\alpha}\right) y(t-\alpha)=\nabla_{a-1}^{\alpha} y(t)$ for $t \in \mathbb{N}_{n+a}$,

(ii) $\left(\Delta_{a}^{-\alpha}\right) y(t+\alpha)=\nabla_{a-1}^{-\alpha} y(t)$ for $t \in \mathbb{N}_{a}$.

Lemma 6 (see [29]). Let $y(t)$ be defined on ${ }_{b+1} \mathbb{N}$. Then the following statements are valid:

(i) $\left.{ }_{b} \Delta^{\alpha}\right) y(t+\alpha)={ }_{b+1} \nabla^{\alpha} y(t)$ for $t \in{ }_{b-n} \mathbb{N}$,

(ii) $\left.{ }_{b} \Delta^{-\alpha}\right) y(t-\alpha)={ }_{b+1} \nabla^{-\alpha} y(t)$ for $t \in{ }_{b} \mathbb{N}$.

If $f(s)$ is defined on $N_{a} \cap{ }_{b} N$ and $a \equiv b(\bmod 1)$ then $(Q f)(s)=f(a+b-s)$. The Q-operator generates a dual identity by which the left type and the right type fractional sums and differences are related. Using the change of variable $u=a+b-s$, in [18] it was shown that

$$
\Delta_{a}^{-\alpha} Q f(t)=Q_{b} \Delta^{-\alpha} f(t),
$$

and, hence,

$$
\Delta_{a}^{\alpha} Q f(t)=\left(Q_{b} \Delta^{\alpha} f\right)(t) .
$$

The proof of (27) follows by (26) and by noting that

$$
-Q \nabla f(t)=\Delta Q f(t) .
$$

Similarly, in the nabla case we have

$$
\nabla_{a}^{-\alpha} Q f(t)=Q_{b} \nabla^{-\alpha} f(t),
$$

and, hence,

$$
\nabla_{a}^{\alpha} Q f(t)=\left(Q_{b} \nabla^{\alpha} f\right)(t) .
$$

The proof of (30) follows by (29) and that

$$
-Q \Delta f(t)=\nabla Q f(t) .
$$

For more details about the discrete version of the Q-operator we refer to [29].

From the difference calculus or time scale calculus, for a natural $n$ and a sequence $f$, we recall

$$
\begin{aligned}
& \Delta^{n} f(t)=\sum_{k=0}^{n}(-1)^{k}\left(\begin{array}{l}
n \\
k
\end{array}\right) f(t+n-k), \\
& \nabla^{n} f(t)=\sum_{k=0}^{n}(-1)^{k}\left(\begin{array}{l}
n \\
k
\end{array}\right) f(t-n+k) .
\end{aligned}
$$

\section{The Fractional Differences and Sums with Binomial Coefficients}

We first give the definition of fractional order of (32) in the left and right sense.

Definition 7. The (binomial) delta left fractional difference and sum of order $\alpha>0$ for a function $f$ defined on $\mathbb{N}_{a}$ are defined by (a)

$B \Delta_{a}^{\alpha}=\sum_{k=0}^{\alpha+t-a}(-1)^{k}\left(\begin{array}{l}\alpha \\ k\end{array}\right) f(t+\alpha-k), \quad t \in \mathbb{N}_{a+n-\alpha}$,

(b)

$B \Delta_{a}^{-\alpha}=\sum_{k=0}^{\alpha+t-a}(-1)^{k}\left(\begin{array}{c}-\alpha \\ k\end{array}\right) f(t-\alpha-k), \quad t \in \mathbb{N}_{a+\alpha}$,

where $(-1)^{k}\left(\begin{array}{c}-\alpha \\ k\end{array}\right)=\left(\begin{array}{c}\alpha+k-1 \\ k\end{array}\right)$.

Definition 8. The (binomial) nabla left fractional difference and sum of order $\alpha>0$ for a function $f$ defined on $\mathbb{N}_{a}$, are defined by

(a)

$$
B \nabla_{a}^{\alpha}=\sum_{k=0}^{t-a-1}(-1)^{k}\left(\begin{array}{l}
\alpha \\
k
\end{array}\right) f(t-k), \quad t \in \mathbb{N}_{a+n},
$$

(b)

$$
B \nabla_{a}^{-\alpha}=\sum_{k=0}^{t-a-1}(-1)^{k}\left(\begin{array}{c}
-\alpha \\
k
\end{array}\right) f(t-k), \quad t \in \mathbb{N}_{a} .
$$

Analogously, in the right case we can define the following.

Definition 9. The (binomial) delta right fractional difference and sum of order $\alpha>0$ for a function $f$ defined on ${ }_{b} \mathbb{N}$ are defined by

(a)

$$
{ }_{b} \Delta B^{\alpha}=\sum_{k=0}^{\alpha+b-t}(-1)^{k}\left(\begin{array}{l}
\alpha \\
k
\end{array}\right) f(t-\alpha+k), \quad t \in b-n+\alpha \mathbb{N},
$$

(b)

$$
{ }_{b} \Delta B^{-\alpha}=\sum_{k=0}^{-\alpha+b-t}(-1)^{k}\left(\begin{array}{c}
-\alpha \\
k
\end{array}\right) f(t+\alpha+k), \quad t \in b-\alpha \mathbb{N} .
$$

Definition 10. The (binomial) nabla right fractional difference and sum of order $\alpha>0$ for a function $f$ defined on ${ }_{b} \mathbb{N}$ are defined by

(a)

$$
{ }_{b} \nabla B^{\alpha}=\sum_{k=0}^{b-t-1}(-1)^{k}\left(\begin{array}{l}
\alpha \\
k
\end{array}\right) f(t-k), \quad t \in_{b-n} \mathbb{N},
$$

(b)

$$
{ }_{b} \nabla B^{-\alpha}=\sum_{k=0}^{b-t-1}(-1)^{k}\left(\begin{array}{c}
-\alpha \\
k
\end{array}\right) f(t-k), \quad t \in{ }_{b} \mathbb{N} .
$$


We next proceed to show that the Riemann fractional differences and sums coincide with the binomial ones defined above. We will use the dual identities in Lemma 5 and Lemma 6, and the action of the discrete version of the Qoperator to follow easy proofs and verifications. In [20], the author used a delta Leibniz's rule to obtain the following alternative definition for Riemann delta left fractional differences:

$$
\begin{array}{r}
\Delta_{a}^{\alpha} f(t)=\frac{1}{\Gamma(-\alpha)} \sum_{s=a}^{t+\alpha}(t-\sigma(s))^{(-\alpha-1)} f(s), \\
\alpha \notin \mathbb{N}, t \in \mathbb{N}_{a+n-\alpha},
\end{array}
$$

then proceeded with long calculations and showed, actually, that

$$
\Delta_{a}^{\alpha} f(t)=B \Delta_{a}^{\alpha} f(t), \quad \Delta_{a}^{-\alpha} f(t)=B \Delta_{a}^{-\alpha} f(t) .
$$

Theorem 11. Let $f$ be defined on suitable domains and $\alpha>0$. Then,

(1)

$$
\Delta_{a}^{\alpha} f(t)=B \Delta_{a}^{\alpha} f(t), \quad \Delta_{a}^{-\alpha} f(t)=B \Delta_{a}^{-\alpha} f(t),
$$

$$
{ }_{b} \Delta^{\alpha} f(t)={ }_{b} \Delta B^{\alpha} f(t), \quad{ }_{b} \Delta^{-\alpha} f(t)=\Delta B_{a}^{-\alpha} f(t),
$$

(3)

$$
\nabla_{a}^{\alpha} f(t)=B \nabla_{a}^{\alpha} f(t), \quad \nabla_{a}^{-\alpha} f(t)=B \nabla_{a}^{-\alpha} f(t),
$$

(4)

$$
{ }_{b} \nabla^{\alpha} f(t)={ }_{b} \nabla B^{\alpha} f(t), \quad{ }_{b} \nabla^{-\alpha} f(t)={ }_{b} \nabla B^{-\alpha} f(t) .
$$

Proof. (1) follows by (42).

(2) By the discrete Q-operator action we have

$$
\begin{aligned}
{ }_{b} \Delta^{\alpha} f(t) & =Q \Delta_{a}(Q f)(t) \\
& =Q \sum_{k=0}^{\alpha+t-a}(-1)^{k}\left(\begin{array}{l}
\alpha \\
k
\end{array}\right)(Q f)(t+\alpha-k) \\
& ={ }_{b} \Delta B^{\alpha} f(t) .
\end{aligned}
$$

The fractional sum part is also done in a similar way by using the Q-operator.

(3) By the dual identity in Lemma 5 (i) and (42), we have

$$
\nabla_{a}^{\alpha} f(t)=\Delta_{a+1}^{\alpha} f(t+\alpha)=B \Delta_{a+1}^{\alpha} f(t+\alpha)=B \nabla_{a}^{\alpha} f(t) .
$$

The fractional sum part can be proved similarly by using Lemma 5 (ii) and (42).

(4) The proof can be achieved by either (2) and Lemma 6 or, alternatively, by (3) and the discrete Q-operator.
Remark 12. In analogous to (41), the authors in [31] used a nabla Leibniz's rule to prove that

$$
\nabla_{a}^{\alpha} f(t)=\frac{1}{\Gamma(-\alpha)} \sum_{s=a+1}^{t}(t-\rho(s))^{\overline{-\alpha-1}} f(s) .
$$

In [30], the authors used a delta Leibniz's Rule to prove the following formula for nabla right fractional differences:

$$
{ }_{b} \nabla^{\alpha} f(t)=\frac{1}{\Gamma(-\alpha)} \sum_{s=t}^{b-1}(s-\rho(t))^{\overline{-\alpha-1}} f(s) .
$$

Similarly, we can use a nabla Leibniz's rule to prove the following formula for the delta right fractional differences:

$$
{ }_{b} \Delta^{\alpha} f(t)=\frac{1}{\Gamma(-\alpha)} \sum_{s=t-\alpha}^{b}(s-\sigma(t))^{(-\alpha-1)} f(s) .
$$

We here remark that the proofs of the last three parts of Theorem 11 can be done alternatively by proceeding as in [20] starting from (49), (50), and (51). Also, it is worth mentioning that mixing both delta and nabla operators in defining delta and nabla right Riemann fractional differences was essential in proceeding, through the dual identities and the discrete Qoperator or delta and nabla type Leibniz's rules, to obtain the main results in this paper [29].

\section{Conclusion}

The impact of fractional calculus in both pure and applied branches of science and engineering started to increase substantially. The main idea of iterating an operator and then generalizing to any order (real or complex) started to be used in the last decade to obtain appropriate discretization for the fractional operators. We mention, from the theory of time scales view point, that how to obtain the fractional operators was a natural question and it was not correlated to the wellknown Grünwald-Letnikov approach. We believe that the discretizations obtained recently in the literature for the fractional operators are different from the one reported within Grünwald-Letnikov method. Bearing all of these thinks in mind we proved that the discrete operators via binomial theorem will lead to the same results as the ones by using the discretization of the Riemann-Liouville operators via time scales techniques. The discrete version of the impressive dual tool Q-operator has been used to prove the equivalency.

\section{References}

[1] S. G. Samko, A. A. Kilbas, and O. I. Marichev, Marichev, Fractional Integrals and Derivatives: Theory and Applications, Gordon and Breach, Yverdon, Switzerland, 1993.

[2] I. Podlubny, Fractional Differential Equations, vol. 198, Academic Press., San Diego, Calif, USA, 1999.

[3] A. A. Kilbas, H. M. Srivastava, and J. J. Trujillo, Theory and Application of Fractional Differential Equations, vol. 204 of NorthHolland Mathematics Studies, Elsevier Science, Amsterdam, The Netherland, 2006. 
[4] B. J. West, M. Bologna, and P. Grigolini, Physics of Fractal Operators, Springer, New York, NY, USA, 2003.

[5] R. L. Magin, Fractional Calculus in Bioengineering, Begell House, West Redding, Conn, USA, 2006.

[6] D. Baleanu, K. Diethelm, E. Scalas, and J. J. Trujillo, Fractional Calculus Models and Numerical Methods, vol. 3 of Series on Complexity, Nonlinearity and Chaos, World Scientific, Hackensack, NJ, USA, 2012.

[7] M. D. Ortigueira, "Fractional central differences and derivatives," Journal of Vibration and Control, vol. 14, no. 9-10, pp. 1255-1266, 2008.

[8] P. Lino and G. Maione, "Tuning PI( $(v)$ fractional order controllers for position control of DC-servomotors," in Proceedings of the IEEE International Symposium on Industrial Electronics (ISIE '10), pp. 359-363, July 2010.

[9] J. A. T. Machado, C. M. Pinto, and A. M. Lopes, "Power law and entropy analysis of catastrophic phenomena," Mathematical Problems in Engineering, vol. 2013, Article ID 562320, 10 pages, 2013.

[10] C. F. Lorenzo, T. T. Hartley, and R. Malti, "Application of the principal fractional metatrigonometric functions for the solution of linear commensurate-order time-invariant fractional differential equations," Philosophical Transactions of the Royal Society A, vol. 371, no. 1990, Article ID 20120151, 2013.

[11] H. L. Gray and N. F. Zhang, "On a new definition of the fractional difference," Mathematics of Computation, vol. 50, no. 182, pp. 513-529, 1988.

[12] K. S. Miller and B. Ross, "Fractional difference calculus," in Proceedings of the International Symposium on Univalent Functions, Fractional Calculus and Their Applications, pp. 139-152, Nihon University, Koriyama, Japan, 1989.

[13] F. M. Atıcı and P. W. Eloe, "A transform method in discrete fractional calculus," International Journal of Difference Equations, vol. 2, no. 2, pp. 165-176, 2007.

[14] F. M. Atıc1 and P. W. Eloe, "Initial value problems in discrete fractional calculus," Proceedings of the American Mathematical Society, vol. 137, no. 3, pp. 981-989, 2009.

[15] F. M. Atıc1 and P. W. Eloe, "Discrete fractional calculus with the nabla operator," Electronic Journal of Qualitative Theory of Differential Equations, no. 3, pp. 1-12, 2009.

[16] F. M. Atıcı and S. Şengül, "Modeling with fractional difference equations," Journal of Mathematical Analysis and Applications, vol. 369, no. 1, pp. 1-9, 2010.

[17] F. M. Atıcı and P. W. Eloe, "Gronwall's inequality on discrete fractional calculus," Computerand Mathematics with Applications, vol. 64, no. 10, pp. 3193-3200, 2012.

[18] T. Abdeljawad, "On Riemann and Caputo fractional differences," Computers and Mathematics with Applications, vol. 62, no. 3, pp. 1602-1611, 2011.

[19] T. Abdeljawad and D. Baleanu, "Fractional differences and integration by parts," Journal of Computational Analysis and Applications, vol. 13, no. 3, pp. 574-582, 2011.

[20] M. Holm, The theory of discrete fractional calculus development and application [dissertation], University of Nebraska, Lincoln, Neb, USA, 2011.

[21] G. A. Anastassiou, "Principles of delta fractional calculus on time scales and inequalities," Mathematical and Computer Modelling, vol. 52, no. 3-4, pp. 556-566, 2010.

[22] G. A. Anastassiou, "Nabla discrete fractional calculus and nabla inequalities," Mathematical and Computer Modelling, vol. 51, no. 5-6, pp. 562-571, 2010.
[23] G. A. Anastassiou, "Foundations of nabla fractional calculus on time scales and inequalities," Computers and Mathematics with Applications, vol. 59, no. 12, pp. 3750-3762, 2010.

[24] N. R. O. Bastos, R. A. C. Ferreira, and D. F. M. Torres, "Discretetime fractional variational problems," Signal Processing, vol. 91, no. 3, pp. 513-524, 2011.

[25] M. Bohner and A. Peterson, Advances in Dynamic Equations on Time Scales, Birkhäuser, Boston, Mass, USA, 2003.

[26] G. Boros and V. Moll, Iresistible Integrals, Symbols, Analysis and Expreiments in the Evaluation of Integrals, Cambridge University Press, Cambridge, UK, 2004.

[27] R. L. Graham, D. E. Knuth, and O. Patashnik, Concrete Mathematics: A Foundation for Copmuter Science, Addison-Wesley, Reading, Mass, USA, 2nd edition, 1994.

[28] J. Spanier and K. B. Oldham, "The pochhammer polynomials $(x)_{n}$," in An Atlas of Functions, pp. 149-156, Hemisphere, Washington, DC, USA, 1987.

[29] T. Abdeljawad, "Dual identities in fractional difference calculus within Riemann," Advances in Difference Equations, vol. 2013, article 36, 2013.

[30] T. Abdeljawad and F. M. Atıc1, "On the definitions of nabla fractional operators," Abstract and Applied Analysis, vol. 2012, Article ID 406757, 13 pages, 2012.

[31] K. Ahrendt, L. Castle, M. Holm, and K. Yochman, "Laplace transforms for the nabla-difference operator and a fractional variation of parameters formula," Communications in Applied Analysis. In press. 


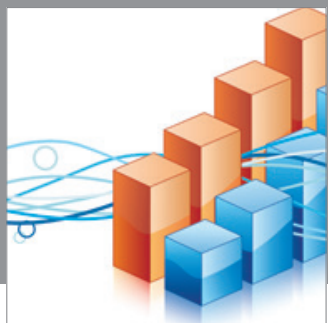

Advances in

Operations Research

mansans

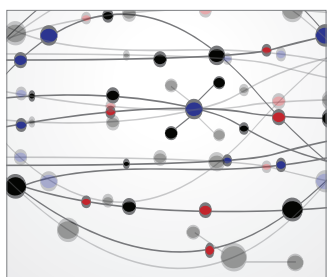

The Scientific World Journal
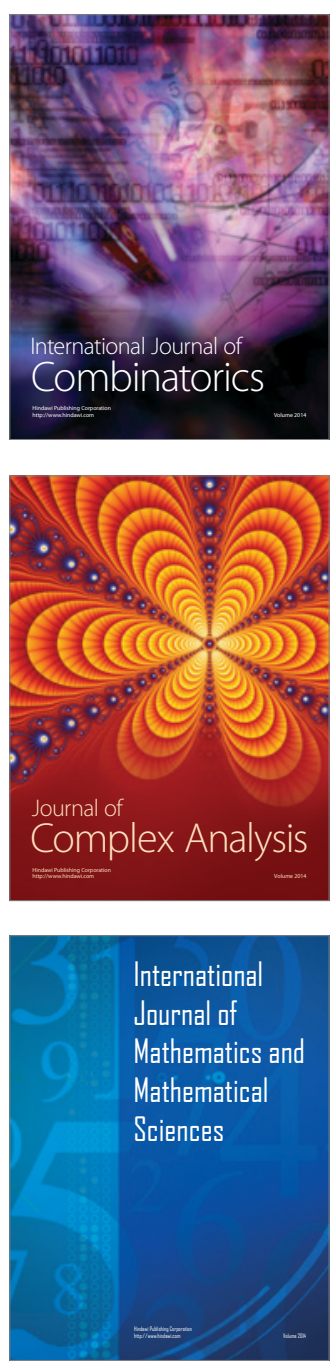
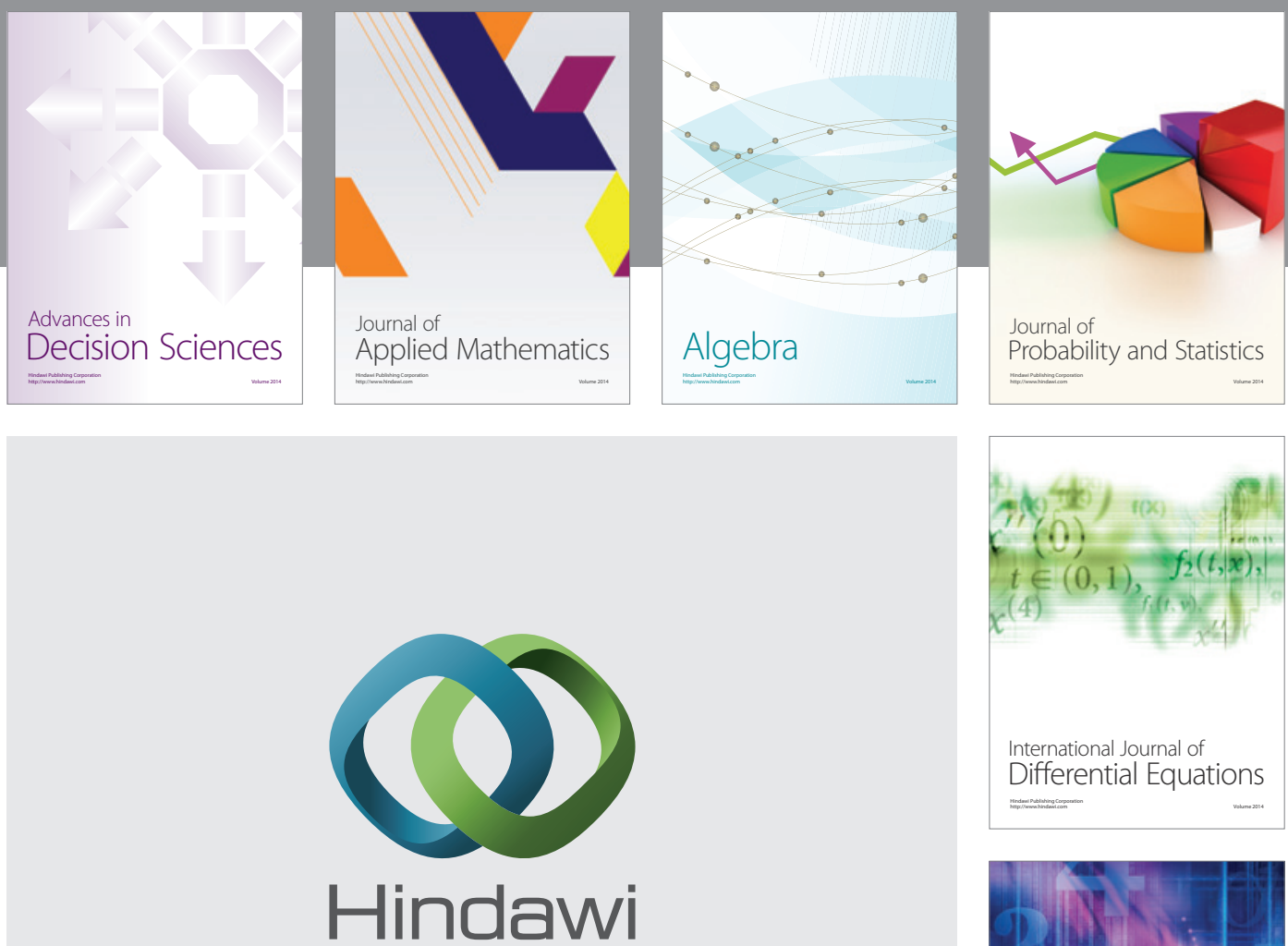

Submit your manuscripts at http://www.hindawi.com
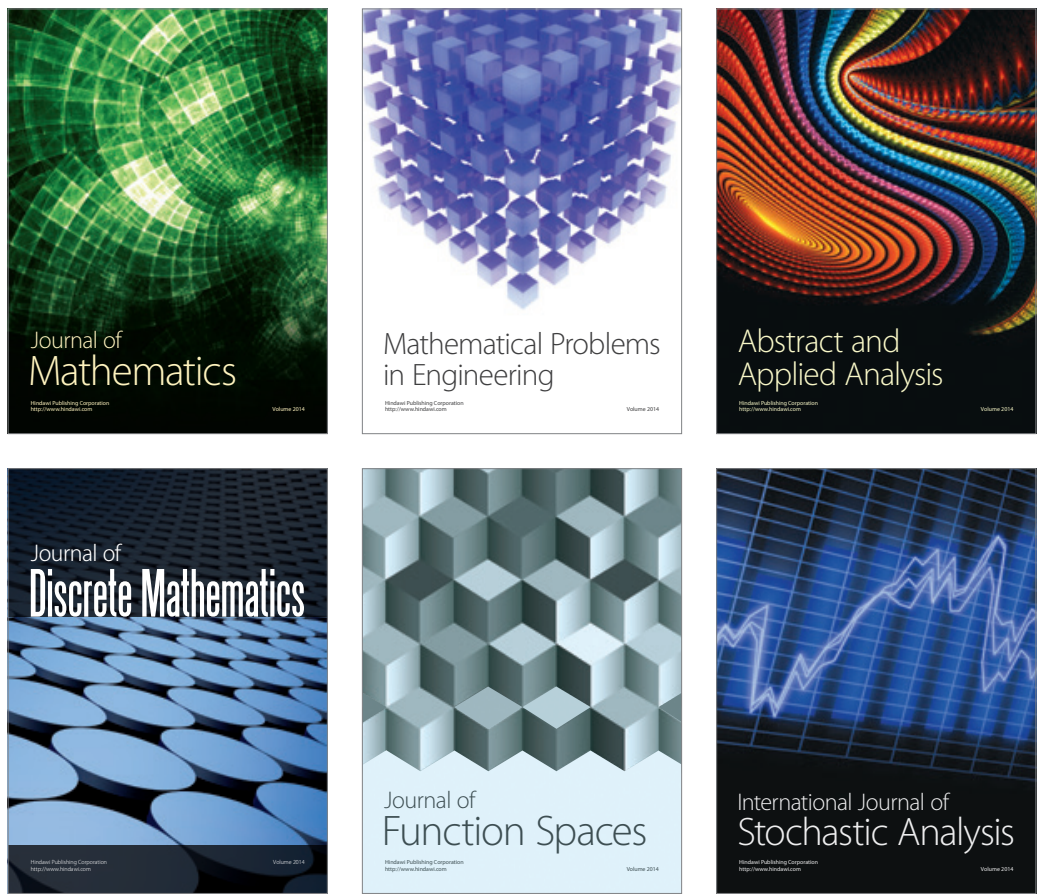

Journal of

Function Spaces

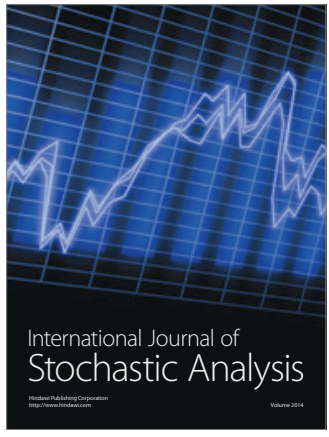

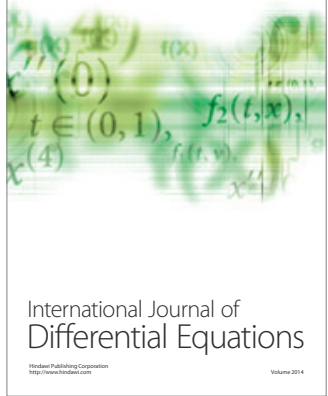
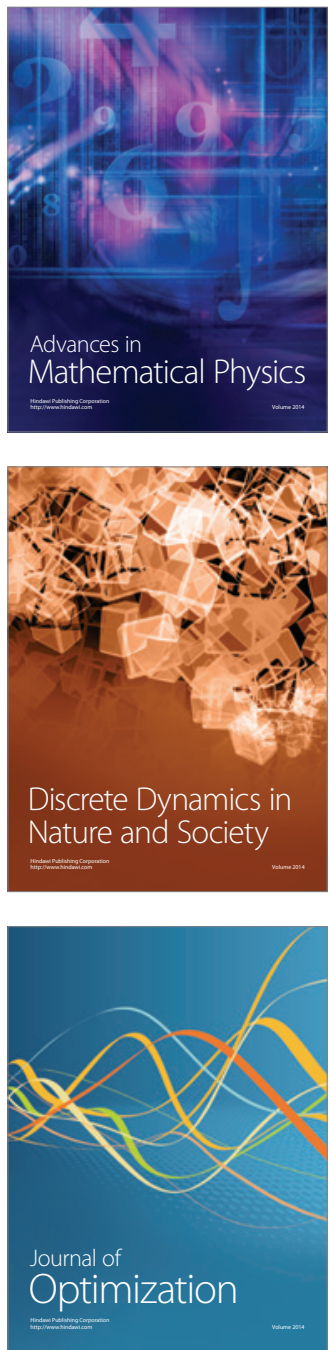